\title{
Reply to the correspondence letter by Meyer et al. 'Complementary and alternative medicine in children'
}

\author{
M. Singendonk • A. Vlieger
}

Received: 24 October 2012 / Accepted: 24 October 2012 /Published online: 8 January 2013

(C) Springer-Verlag Berlin Heidelberg 2012

We appreciate the interest shown in our paper [1] by Dr. Meyer and colleagues. The authors describe in their letter an interesting analysis of all Cochrane reviews in the field of complementary and alternative medicine (CAM) use in children. We agree with the authors that there is a great need for high-quality research in the field of CAM in the pediatric population. The results of this systematic review give insight to the current recommendations on CAM therapy, which is the first step in providing adequate information to parents who would like to visit a CAM therapist with their child or initiate an over-the-counter CAM therapy.

The authors mentioned they found 7 reviews that recommended a certain intervention as well as 14 reviews which concluded that certain interventions should not be performed. We would like to encourage the authors to describe these 21 reviews in more detail in order to help pediatricians and pediatric oncologists provide more specific and evidence-based information to parents. The large number of inconclusive reviews based on the quality of the studies is striking. This underlines our conclusion that adequately conducted and high-quality research of CAM interventions in the pediatric population is highly needed.

\section{Reference}

1. Singendonk M, Kaspers GJ, Naafs-Wilstra M, Meeteren AS, Loeffen J, Vlieger A (2012) High prevalence of complementary and alternative medicine use in the Dutch pediatric oncology population: a multicenter survey. Eur J Pediatr. doi:10.1007/s00431-012-1821-6 